\title{
Remarks on a Class of Nonlinear Schrödinger Equations with Potential Vanishing at Infinity
}

\author{
Hongbo Zhu \\ Faculty of Applied Mathematics, Guangdong University of Technology, Guangzhou 510006, China \\ Correspondence should be addressed to Hongbo Zhu; zhbxw@126.com
}

Received 2 July 2013; Accepted 21 November 2013

Academic Editor: Stepan Agop Tersian

Copyright ( 2013 Hongbo Zhu. This is an open access article distributed under the Creative Commons Attribution License, which permits unrestricted use, distribution, and reproduction in any medium, provided the original work is properly cited.

We study the following nonlinear Schrödinger equation $-\Delta u+V(x) u=K(x) f(u), x \in \mathbb{R}^{N}, u \in H^{1}\left(\mathbb{R}^{N}\right)$, where the potential $V(x)$ vanishes at infinity. Working in weighted Sobolev space, we obtain the ground states of problem $(\mathscr{P})$ under a Nahari type condition. Furthermore, if $V(x), K(x)$ are radically symmetric with respect to $x \in \mathbb{R}^{N}$, it is shown that problem $(\mathscr{P})$ has a positive solution with some more general growth conditions of the nonlinearity. Particularly, if $f(u)=u^{p}$, then the growth restriction $\sigma \leq p \leq N+2 / N-2$ in Ambrosetti et al. (2005) can be relaxed to $\widetilde{\sigma} \leq p \leq N+2 / N-2$, where $\widetilde{\sigma}<\sigma$ if $0<\beta<\alpha<2$.

\section{Introduction}

The motivation of the paper is concerned with the existence of standing waves of the following nonlinear Schrödinger equation:

$$
i \frac{\partial \psi}{\partial t}=-\Delta_{x} \psi+\widetilde{V} \psi-f(x, \psi), \quad(t, x) \in \mathbb{R}_{+} \times \mathbb{R}^{N},
$$

where $i$ is the imaginary unit, $\widetilde{V}$ is a real function on $\mathbb{R}^{N}$, $\psi(x, t): \mathbb{R}^{N} \times[0,+\infty) \rightarrow \mathbb{C}$, and $f$ is supposed to satisfy that $f\left(x, \alpha e^{i \theta}\right)=f(x, \alpha) e^{i \theta}$ for all $\alpha, \theta \in \mathbb{R}$. Problem (1) arises in many applications. For example, in some problems arising in nonlinear optics, in plasma physics, and in condensed matter physics, the presence of many particles leads one to consider nonlinear terms which simulates the interaction effect among them.

For problem (1), we are interested in looking for a stationary solution; that is, $\psi(x, t)=u(x) e^{i \omega t}$ with $u(x)>0$ in $\mathbb{R}^{N}$ and $\omega>0$ (the frequency); then it is not difficult to see that $u$ must satisfy

$$
\begin{gathered}
-\Delta u+V(x) u=f(x, u), \quad x \in \mathbb{R}^{N}, \\
u \in H^{1}\left(\mathbb{R}^{N}\right) .
\end{gathered}
$$

Here and below, $N \geq 3$. Variational approach to (2) was initiated by Rabinowtiz [1], and since then several authors have studied (2) under different assumptions on $V(x)$ and the nonlinearity. If $V(x)$ is positive and bounded away from zero, then, by the well-known concentration compactness principle $[2,3]$, it is shown that there is bound states for problem (2); we mention here the work by Jeanjean and Tanaka [4, 5], Liu and Wang [6], Li et al. [7], Zhu [8], and the references therein.

If the potential $V(x)$ decays to zero at infinity, the methods used in the proceeding papers cannot be employed because the variational theory in $H^{1}\left(\mathbb{R}^{N}\right)$ cannot be used here. The earlier work on (2) we know of where $V(x)$ decays at infinity, is that by Ambrosetti et al. [9]; the authors proved that problem (2) has bounded states for $f(x, u)=K(x) u^{p}$ with

$$
\begin{gathered}
\frac{a}{1+|x|^{\alpha}} \leq V(x) \leq A, \quad 0<K(x) \leq \frac{k}{1+|x|^{\beta}}, \\
\sigma<p<\frac{N+2}{N-2},
\end{gathered}
$$

where

$$
\sigma= \begin{cases}\frac{N+2}{N-2}-\frac{4 \beta}{\alpha(N-2)}, & 0<\beta<\alpha, \\ 1, & \text { otherwise. }\end{cases}
$$

Following [9], by requiring some further assumptions on $V(x), K(x)$, in [10], the authors showed that there exist 
bound states of equation $-\epsilon^{2} \Delta u+V(x) u=K(x) u^{p}, x \in \mathbb{R}^{N}$, $u>0$, for all $p$ satisfying $1<p<(N+2) /(N-2)$, provided that $\epsilon$ is sufficiently small. Motivated by the works $[9,10]$, in paper [11], the authors extended the results to potentials $V(x)$ that can both vanish and decay to zero at infinity. And since then, there are many papers on problem (2) with potential $V(x)$ vanishing at infinity; see, for example, [12-16].

In this paper, more precisely we will focus on the following model equation:

$$
\begin{aligned}
-\Delta u+V(x) u & =K(x) f(u), \quad x \in \mathbb{R}^{N}, \\
u & \in H^{1}\left(\mathbb{R}^{N}\right) .
\end{aligned}
$$

To our best knowledge, it seems that there are few results on problem (5), where $f(u)$ does not satisfy $(A R)$ condition; that is, for some $\theta>0$,

$$
0 \leq F(u) \triangleq \int_{0}^{u} f(t) d t \leq \frac{1}{2+\theta} f(u) u \quad \forall u \in \mathbb{R},
$$

and simultaneously $V(x)$ decays to zero at infinity.

The main aim of the paper is to extend the result of [9] to problem (5) with much more general classes of $f(u)$. Moreover, if $V(x), K(x)$ are radically symmetric with respect to $x \in \mathbb{R}^{N}$, problem (5) will be proved to also have a positive solution with some more general growth conditions of the nonlinearity. Particularly, the result can exactly extend the growth restriction of the special case of $f(u)=u^{p}$ to a new one, and the range of which is bigger than the usual $\sigma \leq p \leq$ $(N+2) /(N-2)$ in [9]; for more details please see Theorem 6 .

Throughout the paper, we make the following assumptions on $V(x), K(x)$, and $f(t)$.

(V) $V: \mathbb{R}^{N} \rightarrow \mathbb{R}$ is continuous and there exist $a, A, \alpha>0$ such that

$$
\frac{a}{1+|x|^{\alpha}} \leq V(x) \leq A .
$$

(K) $K: \mathbb{R}^{N} \rightarrow \mathbb{R}$ is smooth and there exist $\beta, k>0$ such that

$$
0<K(x) \leq \frac{k}{1+|x|^{\beta}}
$$

$\left(F_{1}\right) f_{t}(t)$ is a Caratheodory function and there exists $C>$ 0 such that

$$
\left|f_{t}(t)\right| \leq C\left(1+|t|^{2^{*}-2}\right), \quad \lim _{|t| \rightarrow \infty} \frac{f(t)}{|t|^{2^{*}-1}}=0 .
$$

$\left(F_{2}\right) \lim _{|t| \rightarrow 0}(f(t) /|t|)=0$ and $\lim _{|t| \rightarrow \infty}\left(F(t) / t^{2}\right)=\infty$.

$\left(F_{3}\right) f(t) /|t|$ is nondecreasing in $t \in \mathbb{R} \backslash\{0\}$.

Remark 1. If $\beta>\alpha>0$, then $\liminf _{|x| \rightarrow \infty}(V(x) / K(x))=$ $+\infty$, which implies that $K(x) \leq C V(x)$.
Throughout this paper, we define the following weighted Sobolev space:

$$
\begin{aligned}
H=\left\{u \in \mathscr{D}^{1,2}\left(\mathbb{R}^{N}\right):\right. & \\
& \left.\int_{\mathbb{R}^{N}}\left(|\nabla u|^{2}+V(x)|u|^{2}\right) d x<\infty\right\} .
\end{aligned}
$$

Clearly, $H^{1}\left(\mathbb{R}^{N}\right) \subset H$. $H$ is a Hilbert space with norm and scalar product, respectively,

$$
\begin{gathered}
\|u\|=\left[\int_{\mathbb{R}^{N}}\left(|\nabla u|^{2}+V(x)|u|^{2}\right) d x\right]^{1 / 2}, \\
(u, v)=\int_{\mathbb{R}^{N}}(\nabla u \nabla v+V(x) u v) d x .
\end{gathered}
$$

Denote by $L_{K}^{q}$ the weighted space of measurable $u: \mathbb{R}^{N} \rightarrow \mathbb{R}$ such that

$$
|u|_{q, K}=\left(\int_{\mathbb{R}^{N}} K(x)|u(x)|^{q} d x\right)^{1 / q}<\infty .
$$

$H$ and $L_{K}^{q}$ are particular cases of weighted spaces in discussions in [17], where the following result is proved.

Proposition 2. Let $N \geq 3$, and suppose that $(V),(K)$ hold with $\alpha \in(0,2)$ and $\beta>0$. Then

$$
H \hookrightarrow L_{K}^{p+1},
$$

where $\sigma \leq p \leq(N+2) /(N-2)$, and there is $C>0$ such that

$$
|u|_{p+1, K} \leq C\|u\|, \quad \forall u \in H .
$$

Furthermore, the embedding of $H$ into $L_{K}^{p+1}$ is compact if $p \in$ $(\sigma,(N+2) /(N-2))$.

Remark 3. For the case of $\beta \geq \alpha>0, \sigma=1$, and consequently $H \hookrightarrow L_{K}^{p+1}$, for all1 $\leq p \leq(N+2) /(N-2)$, and also the embedding is compact if $1<p<(N+2) /(N-2)$.

Furthermore, we define the energy functional associated with problem (5) by

$$
\begin{aligned}
I(u)= & \frac{1}{2} \int_{\mathbb{R}^{N}}\left(|\nabla u|^{2}+V(x)|u|^{2}\right) d x \\
& -\int_{\mathbb{R}^{N}} K(x) F(u) d x, \\
F(u)= & \int_{0}^{u} f(t) d t .
\end{aligned}
$$

By Proposition 2, $I$ is well defined on $H$ and $I \in C^{1}(H, \mathbb{R})$ with

$$
\begin{aligned}
\left\langle I^{\prime}(u), v\right\rangle= & \int_{\mathbb{R}^{N}}(\nabla u \nabla v+V(x) u v) d x \\
& -\int_{\mathbb{R}^{N}} K(x) f(u) v d x \quad \forall v \in H .
\end{aligned}
$$


Definition 4. A function $u \in H$ is said to be a solution of problem (5) provided that $u \neq \equiv 0$ satisfies

$$
\begin{aligned}
\int_{\mathbb{R}^{N}}(\nabla u \nabla \varphi+V(x) u \varphi) d x \\
-\int_{\mathbb{R}^{N}} K(x) f(u) \varphi d x=0 \quad \forall \varphi \in H .
\end{aligned}
$$

Notation. Hereafter we use the following notation.

(i) $2^{*}=2 N / N-2$ if $N \geq 3$ and for some $2<q<2^{*}=\infty$ if $N=2$.

(ii) $L^{q}(\Omega), 1 \leq q \leq+\infty, \Omega \subseteq \mathbb{R}^{N}$, denotes a Lebesgue space; the norm in $L^{q}(\Omega)$ is denoted by $|u|_{q, \Omega}$.

(iii) For any $R>0$ and for any $y \in \mathbb{R}^{N}, B_{R}(y)$ denotes the ball of radius $R$ centered at $y$.

(iv) $C, C_{i}$ are various positive constants.

(v) $o$ (1) denotes the quantity which tends to zero as $n \rightarrow$ $\infty$.

Our main results are the following.

Theorem 5. Let $\left(F_{1}\right)-\left(F_{3}\right)$ and $(V),(K)$ hold with $\beta>\alpha, \alpha \in$ $(0,2)$. Then problem (5) has a ground state $u \in H^{1}\left(\mathbb{R}^{N}\right)$.

If $V(x), K(x)$ are radically symmetric with respect to $x \in$ $\mathbb{R}^{N}$ and $f(t)$ satisfies the following conditions:

$\left(F_{4}\right) f \in C\left(\mathbb{R}, \mathbb{R}^{+}\right), f(t) \equiv 0$ for all $t \leq 0 ;$

$\left(F_{5}\right) f(t) / t$ is nondecreasing with respect to $t>0$;

$\left(F_{6}\right) \lim _{t \rightarrow 0}\left(f(t) / t^{p}\right)=l_{1} \in[0, \infty) ; \lim _{t \rightarrow \infty}\left(f(t) / t^{q}\right)=$ $l_{2} \in(0, \infty)$, where $q^{*}-1<p \leq q<N+2 / N-2$, and

$$
q^{*}= \begin{cases}\frac{4(N-1-\beta)+2 \alpha}{2(N-1)-\alpha}, & 0<\beta<\alpha<2 \\ 2, & \text { otherwise }\end{cases}
$$

then we have the following.

Theorem 6. Assume that $V(x), K(x)$ are radically symmetric with respect to $x \in \mathbb{R}^{N}$, and let $(V),(K)$, and $\left(F_{4}\right)-\left(F_{6}\right)$ hold. Then problem (5) has a positive solution $u \in H_{r}^{1}\left(\mathbb{R}^{N}\right)$.

Remark 7. If $f(u)=u^{k}$, then the growth restriction $\sigma \leq k \leq$ $N+2 / N-2$ in [9] can be relaxed to $q^{*}-1 \leq k \leq N+2 / N-2$, where $q^{*}-1<\sigma$ if $0<\beta<\alpha<2$.

\section{Variational Setting and Some Preliminaries}

In this section, we describe the variational framework for the study of the critical points of the functional defined in (14). Set

$$
c:=\inf \{I(u): u \in H\} \text {, }
$$

where $\mathcal{N}=\left\{u \in H \backslash\{0\}: \gamma(u) \triangleq\left\langle I^{\prime}(u), u\right\rangle=0\right\}$. First, it is necessary to show that $c$ is a positive number. Now we give the following two lemmas.
Lemma 8. Suppose that $\left(F_{1}\right)-\left(F_{3}\right)$ hold. Then for each $u \in H \backslash$ $\{0\}$, there exists a unique $t=t(u)>0$ such that $t(u) u \in \mathcal{N}$ and $\max _{t \geq 0} I(t u)=I(t(u) u)$.

Proof. The proof of this lemma is similar to the case of assuming $(A R)$ condition, which can be found in [18], so we omit it here.

Lemma 9. $c>0$

Proof. Let

$$
c_{1}:=\inf _{u \in \mathscr{H} \backslash\{0\}} \max _{t \geq 0} I(t u), \quad c_{2}:=\inf _{\gamma \in \Gamma t \in[0,1]} \max _{t} I(\gamma(t)),
$$

where $\Gamma:=\{\gamma \in C(([0,1]), H): \gamma(0)=0, I(\gamma(1))<0\}$. By Lemma 8, it is easy to see $c=c_{1}$.

For any $u \in H \backslash\{0\}$, by $\left(F_{2}\right)$, there exists $t>0$ large enough such that $I(t u)<0$. Let $\widetilde{\gamma}(s)=s t u$; then $\widetilde{\gamma}(s) \in \Gamma$, and

$$
c_{2} \leq I(\tilde{\gamma}(s))=I(s t u) \leq \max _{t \geq 0} I(t u),
$$

so $c_{1} \geq c_{2}$. From $\left(F_{1}\right),\left(F_{2}\right)$, there is $\rho>0$ small enough such that $\inf _{\|u\|=\rho} I(u)>\alpha>0$. Since $\widetilde{\gamma}(0)=0$, for large enough $t>0,\|\tilde{\gamma}(1)\|>\rho$, there exists $s_{0} \in[0,1]$ such that $\left\|\widetilde{\gamma}\left(s_{0}\right)\right\|=\rho$, so

$$
c_{2} \geq I\left(\tilde{\gamma}\left(s_{0}\right)\right) \geq \inf _{\|u\|=\rho} I(u)>\alpha>0 .
$$

Thus, $c=c_{1} \geq c_{2}>0$.

Lemma 10. Let $\left\{u_{n}\right\}$ be a minimizing sequence of $c$ defined in (18). Then

(i) there is $\rho>0$ such that $\liminf _{n \rightarrow \infty}\left\|u_{n}\right\| \geq \rho>0$,

(ii) $\left\{u_{n}\right\}$ is bounded in $H$,

(iii) for a subsequence, $\left\{u_{n}\right\}$ converges weakly to $u \neq \equiv 0$.

Proof. (i) By $\left(F_{1}\right),\left(F_{2}\right)$, for any $\epsilon>0$, there exists $C_{\epsilon}>0$ such that

$$
|f(t)| \leq \epsilon\left(|t|+|t|^{2^{*}-1}\right)+C_{\epsilon}|t|^{q-1}, \quad q \in\left(2,2^{*}\right) .
$$

From $\gamma\left(u_{n}\right)=0, H \hookrightarrow L_{K}^{q}\left(\mathbb{R}^{N}\right)$, it is easy to get that $\liminf _{n \rightarrow \infty}\left\|u_{n}\right\| \geq \rho>0$.

(ii) If $\left\{u_{n}\right\}$ is not bounded, we define $v_{n}=u_{n} /\left\|u_{n}\right\|$, so $\left\|v_{n}\right\|=1$. Passing to a subsequence we have

$$
\begin{aligned}
& v_{n} \rightarrow v \in H, \quad v_{n} \longrightarrow v \in L_{K}^{q}\left(\mathbb{R}^{N}\right), \\
& 2<q<2^{*}, \quad v_{n} \longrightarrow v \quad \text { a.e. on } \mathbb{R}^{N} .
\end{aligned}
$$

If $v \not \equiv 0$, we have

$$
\frac{1}{2}-\int_{\mathbb{R}^{N}} K(x) \frac{F\left(u_{n}\right)}{\left|u_{n}\right|^{2}}\left|v_{n}\right|^{2} d x=\frac{c+o(1)}{\left\|u_{n}\right\|^{2}}>0 .
$$

By Fatou's lemma and $\left(F_{2}\right)$, we have a contradiction as follows:

$$
\begin{aligned}
\frac{1}{2} & \geq \liminf _{n \rightarrow \infty} \int_{\mathbb{R}^{N}} K(x) \frac{F\left(u_{n}\right)}{\left|u_{n}\right|^{2}}\left|v_{n}\right|^{2} d x \\
& \geq \int_{\mathbb{R}^{N}} \liminf _{n \rightarrow \infty} K(x) \frac{F\left(u_{n}\right)}{\left|u_{n}\right|^{2}}\left|v_{n}\right|^{2} d x=\infty .
\end{aligned}
$$


If $v \equiv 0$, by Proposition $2, v_{n} \longrightarrow 0$ in $L_{K}^{q}\left(\mathbb{R}^{N}\right)$. By (22) and for any $R>0$ which is determined later, we get

$$
\int_{\mathbb{R}^{N}} K(x) F\left(R v_{n}\right) d x \longrightarrow 0 \text { as } n \longrightarrow \infty .
$$

By Lemma $8, I\left(t u_{n}\right) \leq I\left(u_{n}\right)$ for any $t>0$; then we have

$$
\begin{aligned}
c+ & o(1) \longleftarrow I\left(u_{n}\right) \\
& \geq I\left(R v_{n}\right)=\frac{1}{2} R^{2}-\int_{\mathbb{R}^{N}} K(x) F\left(R v_{n}\right) \longrightarrow \frac{1}{2} R^{2}-o(1),
\end{aligned}
$$

which is a contradiction if we take $R>(2 c)^{1 / 2}$. Thus $\left\{u_{n}\right\}$ is bounded in $H$.

(iii) We can assume $\left\{u_{n}\right\}$ weakly converges to $u$. If $u \equiv 0$, then by (i) and noting the fact that $H \hookrightarrow L_{K}^{q}, q \in\left(2,2^{*}\right)$ is compact, we have a contradiction as follows:

$$
0<\rho^{2} \leq\left\|u_{n}\right\|^{2}=\int_{\mathbb{R}^{N}} K(x) f\left(u_{n}\right) u_{n} d x \longrightarrow 0 .
$$

\section{Proof of the Main Results}

The aim of this section is to prove Theorems 5 and 6 . For Theorem 5, we will take two steps; the first is to show the existence of nonzero critical point $u \in H$ of $I$, and the second is to prove the critical point $u$ is a bound state; that is $u \in L^{2}\left(\mathbb{R}^{N}\right)$. To prove Theorem 6, we use a weighted Sobolev embedding theorem, which is based upon the results discussed in [19].

\section{Proof of Theorem 5}

Step 1. Let $\left\{u_{n}\right\} \subset \mathcal{N}$ be the sequence minimizing for $c$ given in (18). By Lemma 10, $\left\{u_{n}\right\}$ is bounded in $H$, and there is some $0 \not \equiv u \in H$ such that

$$
u_{n} \stackrel{n}{\rightarrow} u \quad \text { weakly in } H,
$$

and by Proposition 2 and Remark 3, we get $u_{n} \longrightarrow u$ in $L_{K}^{q}\left(\mathbb{R}^{N}\right), q \in\left(2,2^{*}\right)$, and consequently

$$
\begin{array}{r}
\int_{\mathbb{R}^{N}} K(x) F\left(u_{n}\right) d x \longrightarrow \int_{\mathbb{R}^{N}} K(x) F(u) d x \\
\text { as } n \longrightarrow \infty .
\end{array}
$$

Using the lower semicontinuity, we have $I(u) \leq c$. If $u \in \mathcal{N}$, we have $I(u)=c$. If $u \notin \mathcal{N}$, by Lemma 8 , there exists $t=$ $t(u)>0$ such that $t(u) u \in \mathcal{N}$; then

$$
\begin{aligned}
c & \leq I(t(u) u) \\
& \leq \liminf _{n \rightarrow \infty} I\left(t(u) u_{n}\right) \\
& \leq \liminf _{n \rightarrow \infty} I\left(u_{n}\right)=c .
\end{aligned}
$$

Since $\mathcal{N}$ is smooth, the minimizer is a critical point of $I$.

Next we will show that the solution found above in $H$ belongs indeed to $L^{2}\left(\mathbb{R}^{N}\right)$. For this purpose, we require some preliminary decay estimates on $u$, which are essentially motivated by [9]. However, we should mention that these proofs are partly different from those in [9]. First we will give the following proposition, which is a special case of Proposition 11 in [9].

Proposition 11. Let $0<\alpha<2$ and $2<q<2^{*}$. Then for all $\epsilon>0$ there exists $\bar{R}>0$ such that for all $R>\bar{R}$

$$
\begin{aligned}
& \int_{|x|>R} K(x)|u(x)|^{q} d x \\
& \quad \leq \epsilon\left(\int_{|x|>R}\left[|\nabla u(x)|^{2}+V(x) u^{2}(x)\right] d x\right)^{q / 2},
\end{aligned}
$$

$\forall u \in H$

Lemma 12. Suppose that $(V),(K),\left(F_{1}\right)$, and $\left(F_{2}\right)$ hold. Let $\beta>$ $\alpha, \alpha \in(0,2)$ and let $u$ be the critical point of I on $H$. Then there exist $t_{0}, \delta_{1} \in(0,1)$ and $n(\alpha)>0$ such that, for all $n \geq n(\alpha)$,

$$
\begin{aligned}
& \int_{\Omega_{n+1}}\left(|\nabla u|^{2}+V(x)|u|^{2}\right) d x \\
& \leq {\left[\left(1-t_{0}\right)+\delta_{1} t_{0}\right] } \\
& \quad \times \int_{\Omega_{n}}\left(|\nabla u|^{2}+V(x)|u|^{2}\right) d x
\end{aligned}
$$

where

$$
\Omega_{n}=\mathbb{R}^{N} \backslash B_{R_{n}}, \quad R_{n}=b^{n}, \quad b=\left(\frac{1}{1-t_{0}}\right)^{1 / 4} .
$$

Proof. Since $\beta>\alpha$, there is $\delta \in(0,1)$ such that $\beta>\alpha+\delta$. Let $\gamma=\alpha+\delta$; then $\gamma \in(0,3)$, so there exists $\delta_{1} \in(0,1)$ such that $(\gamma+1) / 4<1-\delta_{1}$.

Noting that

$$
\lim _{t \rightarrow 0^{+}} \frac{\ln \left[(1-t)+\delta_{1} t\right]}{(1 / 4) \ln (1 /(1-t))}=-4\left(1-\delta_{1}\right)<-(\gamma+1),
$$

we may choose $t_{0} \in(0,1)$ such that

$$
\frac{\ln \left[\left(1-t_{0}\right)+\delta_{1} t_{0}\right]}{(1 / 4) \ln \left(1 /\left(1-t_{0}\right)\right)}<-(\gamma+1) \text {. }
$$

Let $R_{n}$ be given by (34), and let $\chi_{n}(x)$ be a cut-off function such that

$$
\chi_{n}(x)= \begin{cases}0, & \text { if } 0 \leq|x| \leq R_{n} \\ 1, & \text { if }|x| \geq R_{n+1} .\end{cases}
$$

Moreover, there exists $A_{0}>0$, independent of $n$, such that $\left|\nabla \chi_{n}(x)\right| \leq A_{0} /\left(R_{n+1}-R_{n}\right)$ for all $x \in \mathbb{R}^{N}$. Noting that $\alpha \in$ $(0,2)$ and $b>1$, we see that

$$
\lim _{n \rightarrow \infty} \frac{1+b^{\alpha(n+1)}}{b^{2(n+1)}}\left(\frac{A_{0} b}{b-1}\right)^{2}=0 .
$$

So there exists $n(\alpha)>0$ such that, for all $n \geq n(\alpha)$,

$$
\frac{1+b^{\alpha(n+1)}}{b^{2(n+1)}}\left(\frac{A_{0} b}{b-1}\right)^{2} \leq\left(2 \delta_{1} t_{0}\right)^{2} a,
$$


where $a$ is given by $(V)$. From (39) and (V), for $R_{n} \leq|x| \leq$ $R_{n+1}$, we have

$$
\begin{aligned}
\left|\nabla \chi_{n}(x)\right|^{2} & \leq A_{0}^{2}\left|R_{n+1}-R_{n}\right|^{-2} \\
& =b^{-2(n+1)}\left(\frac{A_{0} b}{b-1}\right)^{2} \\
& \leq \frac{a}{1+\left(R_{n+1}\right)^{\alpha}}\left[2 \delta\left(1-\eta_{0}\right)\right]^{2} \\
& \leq V(x)\left(2 \delta_{1} t_{0}\right)^{2} .
\end{aligned}
$$

Then by the definition of $\chi_{n}(x)$, for all $n \geq n(\alpha)$ and $x \in \mathbb{R}^{N}$,

$$
\left|\nabla \chi_{n}(x)\right|^{2} \leq V(x)\left(2 \delta_{1} t_{0}\right)^{2} .
$$

Since $\left\langle I^{\prime}(u), u \chi_{n}\right\rangle=0$, by $\left(F_{1}\right),\left(F_{2}\right)$, and Young inequality, for any $\epsilon>0, \epsilon_{1}>0$, we have

$$
\begin{aligned}
& \int_{\mathbb{R}^{N}} \chi_{n}\left(|\nabla u|^{2}+V(x)|u|^{2}\right) d x \\
& =\int_{\mathbb{R}^{N}} K(x) f(u) u \chi_{n} d x-\int_{\mathbb{R}^{N}} \nabla u \nabla \chi_{n} u d x \\
& \leq \int_{\Omega_{n}} K(x)\left[\epsilon\left(|u|^{2}+|u|^{2^{*}}\right)+C_{\epsilon}|u|^{q}\right] d x \\
& \quad+\int_{\Omega_{n}} \epsilon_{1}|\nabla u|^{2} d x+\int_{\Omega_{n}} \frac{1}{4 \epsilon_{1}}\left|\nabla \chi_{n}\right|^{2}|u|^{2} d x,
\end{aligned}
$$

where $q \in\left(2,2^{*}\right)$. Taking $\epsilon_{1}=\delta_{1} t_{0}$, then by (41), (42), for all $n \geq n(\alpha)$,

$$
\begin{aligned}
& \int_{\Omega_{n+1}}\left(|\nabla u|^{2}+V(x)|u|^{2}\right) d x \\
& \leq \int_{\Omega_{n}} \chi_{n}\left[|\nabla u|^{2}+V(x)|u|^{2}\right] d x \\
& \leq \int_{\Omega_{n}} K(x)\left[\epsilon\left(|u|^{2}+|u|^{2^{*}}\right)+C_{\epsilon}|u|^{q}\right] d x \\
& \quad+\delta_{1} t_{0} \int_{\Omega_{n}}\left[|\nabla u|^{2}+V(x)|u|^{2}\right] d x .
\end{aligned}
$$

Suppose that $\|u\|^{2} \leq \Gamma, \Gamma>0$; then, from Proposition 11 and Remark 3, for the above $\epsilon>0$ given and $R$ sufficiently large, we deduce that

$$
\begin{aligned}
& \int_{\Omega_{n+1}}\left(|\nabla u|^{2}+V(x)|u|^{2}\right) d x \\
& \leq\left[\left(\epsilon+\epsilon \Gamma^{\left(2^{*}-2\right) / 2}+\epsilon C_{\epsilon} \Gamma^{(q-2) / 2}\right)+\delta_{1} t_{0}\right] \\
& \quad \times \int_{\Omega_{n}}\left[|\nabla u|^{2}+V(x)|u|^{2}\right] d x .
\end{aligned}
$$

Choosing sufficiently small $\epsilon>0$ (and hence for $R$ large) such that $\epsilon+\epsilon \Gamma^{\left(2^{*}-2\right) / 2}+\epsilon C_{\epsilon} \Gamma^{(q-2) / 2}<1-t_{0}$, we obtain the assertion.

Lemma 13. Let $n(\alpha)$ be given in Lemma 12. Then there exists $R(\alpha)>n(\alpha)$ such that, for any $\rho>R(\alpha)$,

$$
\begin{aligned}
& \int_{|x|>\rho}\left(|\nabla u|^{2}+V(x)|u|^{2}\right) d x \\
& \quad \leq\|u\|^{2} \exp \left\{C\left(\delta_{1}, \eta_{0}, \theta\right) \ln \rho\right\},
\end{aligned}
$$

where $C\left(\delta_{1}, t_{0}, \theta\right)<-(\gamma+1)$.

Proof. From (36), there is $\theta \in(0,1)$ such that

$$
C\left(\delta_{1}, t_{0}, \theta\right):=\theta \frac{\ln \left[\left(1-t_{0}\right)+\delta_{1} t_{0}\right]}{(1 / 4) \ln \left(1 /\left(1-t_{0}\right)\right)}<-(\gamma+1) .
$$

Since

$$
\frac{\lim [((\ln R-\ln n(\alpha)) / \ln b)-2]}{(\theta \ln R) / \ln b}=\frac{1}{\theta}>1 .
$$

There exists a constant $R(\alpha)>n(\alpha)$ such that

$$
\begin{gathered}
\ln R(\alpha)-\ln n(\alpha)>2 \ln b, \\
\frac{\ln \rho-\ln n(\alpha)}{\ln b}-2 \geq \frac{\theta \ln \rho}{\ln b}>0, \quad \forall \rho>R(\alpha) .
\end{gathered}
$$

Given $\rho>R(\alpha)$, there exist two integers $\widetilde{n}, \bar{n}$ such that

$$
R_{\bar{n}} \leq n(\alpha) \leq R_{\bar{n}+1}, \quad R_{\widetilde{n}-1} \leq \rho \leq R_{\tilde{n}}
$$

Thus

$$
\widetilde{n}-\bar{n}>\frac{\ln \rho-\ln n(\alpha)}{\ln b}>2 .
$$

By Lemma 12, (49), and (51), we deduce that

$$
\begin{aligned}
\int_{|x|>\rho} & \left(|\nabla u|^{2}+V(x)|u|^{2}\right) d x \\
\leq & \int_{|x|>R_{\tilde{n}-1}}\left(|\nabla u|^{2}+V(x)|u|^{2}\right) d x \\
\leq & {\left[\left(1-t_{0}\right)+\delta_{1} t_{0}\right]^{\tilde{n}-\bar{n}-2} } \\
& \times \int_{|x|>R_{\bar{n}+1}}\left[|\nabla u|^{2}+V(x)|u|^{2}\right] d x \\
\leq & \|u\|^{2} \exp \left\{\ln \left[\left(1-t_{0}\right)+\delta_{1} t_{0}\right]\left(\frac{\ln \rho-\ln n(\alpha)}{\ln b}-2\right)\right\} \\
\leq & \|u\|^{2} \exp \left\{C\left(\delta_{1}, t_{0}, \theta\right) \ln \rho\right\} .
\end{aligned}
$$

That is, (45) holds. 
Proof of Theorem 5

Step 2. Let $u$ be the weak solution found in $H$, and let $r \geq 2$, and $|y| \geq 2 r$; then

$$
\begin{aligned}
\sup & \left\{\frac{1+|x|^{\gamma}}{a|y|^{\gamma}}: x \in B_{r}(y)\right\} \\
& \leq \frac{1+(r+|y|)^{\gamma}}{a|y|^{\gamma}} \\
& \leq \sup \left\{\frac{1+((3 / 2)|y|)^{\gamma}}{a|y|^{\gamma}}:|y| \geq 4\right\},
\end{aligned}
$$

where $\gamma=\alpha+\delta_{1}$. Denote

$$
C_{1}(\gamma)=\sup \left\{\frac{1+((3 / 2)|y|)^{\gamma}}{a|y|^{\gamma}}:|y| \geq 4\right\}=\frac{1}{4^{\gamma} a}+\frac{3^{\gamma}}{2^{\gamma} a} .
$$

Then

$$
\sup \left\{\frac{1+|x|^{\gamma}}{a}: x \in B_{r}(y)\right\} \leq C_{1}(\gamma)|y|^{\gamma}
$$

Since $B_{r}(y) \subset\left\{x \in \mathbb{R}^{N}:|x| \geq|y| / 2\right\}, \gamma>\alpha$, by Lemma 13 and (55), for all $|y| \geq 2 R(\alpha)$, we have

$$
\begin{aligned}
\int_{B_{r}(y)} u^{2} d x & \leq \int_{B_{r}(y)} \frac{1+|x|^{\gamma}}{a} \frac{1+|x|^{\alpha}}{1+|x|^{\gamma}} V(x) u^{2} d x \\
& \leq C_{1}(\gamma)|y|^{\gamma} \int_{B_{r}(y)} V(x) u^{2} d x \\
& \leq C_{1}(\gamma)|y|^{\gamma} \int_{|x| \geq|y| / 2} V(x) u^{2} d x \\
& \leq C_{1}(\gamma) 2^{-C\left(\delta_{1}, t_{0}, \theta\right)}|y|^{\gamma}\|u\|^{2}|y|^{C\left(\delta_{1}, t_{0}, \theta\right)} \\
& =C_{2}\|u\|^{2}|y|^{\gamma+C\left(\delta_{1}, t_{0}, \theta\right)}
\end{aligned}
$$

where $C_{2}=C_{1}(\gamma) 2^{-C\left(\delta_{1}, t_{0}, \theta\right)}$.

With similar arguments of [15], let $m \in \mathbb{N}^{+}$with $\left|y_{i}\right| \geq$ $2(i=1 \cdots m)$ be such that

$$
B_{5} \backslash B_{2} \subset \bigcup_{i=1}^{m} B_{1}\left(y_{i}\right) \text {. }
$$

Let $K_{0}$ denote a positive integral such that $2^{K_{0}}>R(\alpha)$; by (56) we deduce that

$$
\begin{aligned}
\int_{|x| \geq 2} u^{2} d x \leq & \sum_{k=0}^{\infty} \int_{2^{k}\left(B_{5} \backslash B_{2}\right)} u^{2} d x \\
\leq & \sum_{i=1}^{m} \sum_{k=0}^{\infty} \int_{B_{2^{k}}\left(2^{k} y_{i}\right)} u^{2} d x \\
\leq & \sum_{i=1}^{m} \sum_{k=0}^{K_{0}-1} \int_{B_{2^{k}}\left(2^{k} y_{i}\right)} u^{2} d x \\
& +C_{2}\|u\|^{2} \sum_{i=1}^{m} \sum_{k=K_{0}}^{\infty}\left|2^{k} y_{i}\right|^{\gamma+C\left(\delta_{1}, t_{0}, \theta\right)} .
\end{aligned}
$$

Since $\gamma+C\left(\delta_{1}, t_{0}, \theta\right)<-1$, then the above inequality implies that $\int_{|x| \geq 2} u^{2} d x<+\infty$ and $u \in L^{2}\left(\mathbb{R}^{N}\right)$. Thus, $u \in H^{1}\left(\mathbb{R}^{N}\right)$ is a bound state of problem (5).

Before the proof of Theorem 6, let us introduce some functional spaces. Denote by $\mathscr{H}$ the weighted Sobolev space

$$
\mathscr{H}:=\left\{D_{r}^{1,2}\left(\mathbb{R}^{N}\right): \int_{\mathbb{R}^{N}}\left(|\nabla u|^{2}+V(|x|) u^{2}\right) d x<\infty\right\},
$$

where $\mathscr{D}_{r}^{1,2}=\left\{u \in L^{2^{*}}\left(\mathbb{R}^{N}\right): u(x)=u(|x|), \nabla u \in L^{2}\left(\mathbb{R}^{N}\right)\right\}$.

Denote by $\mathscr{L}_{K}^{q}$ the weighted space of measurable $u$ : $\mathbb{R}^{N} \longrightarrow \mathbb{R}$ such that

$$
|u|_{q, K}=\left(\int_{\mathbb{R}^{N}} K(|x|)|u(x)|^{q} d x\right)^{1 / q}<\infty .
$$

Now we give the following embedding result, whose proof can be derived from Theorem 1 of [19].

Proposition 14. Let $N \geq 3$, and suppose that $V(x), K(x)$ are radial, and the assumptions $(V),(K)$ hold with $\alpha \in(0,2)$ and $\beta>0$. Then

$$
\mathscr{H} \hookrightarrow \mathscr{L}_{K}^{q},
$$

where $q^{*} \leq q \leq 2 N /(N-2)$. Furthermore, the embedding is compact if $q \in\left(q^{*}, 2 N /(N-2)\right)$.

Proof of Theorem 6. Based upon Proposition 14, the proof of Theorem 6 can be followed from some standard techniques; we leave the details to the readers.

\section{Acknowledgment}

This work was supported by NSFC Grant no. 11201083.

\section{References}

[1] P. H. Rabinowitz, "On a class of nonlinear Schrödinger equations," Zeitschrift für Angewandte Mathematik und Physik, vol. 43, no. 2, pp. 270-291, 1992.

[2] P. L. Lions, "The concentration-compactness in the calculus of variation. The locally compact case, Part I," Annales de l'Institut Henri Poincare (C) Non Linear Analysis, vol. 1, pp. 109-145, 1984.

[3] P. L. Lions, "The concentration-compactness in the calculus of variation. The locally compact case, PartII," Annales de l'Institut Henri Poincare (C) Non Linear Analysis, vol. 1, pp. 223-283, 1984.

[4] L. Jeanjean and K. Tanaka, "A positive solution for an asymptotically linear elliptic problem on $\mathbb{R}^{N}$ autonomous at infinity," ESAIM. Control, Optimisation and Calculus of Variations, vol. 7, pp. 597-614, 2002.

[5] L. Jeanjean and K. Tanaka, "A positive solution for a nonlinear Schrödinger equation on $\mathbb{R}^{N}$," Indiana University Mathematics Journal, vol. 54, no. 2, pp. 443-464, 2005.

[6] Z. Liu and Z.-Q. Wang, "Existence of a positive solution of an elliptic equation on $\mathbb{R}^{N}$," Proceedings of the Royal Society of Edinburgh A, vol. 134, no. 1, pp. 191-200, 2004.

[7] Y. Li, Z.-Q. Wang, and J. Zeng, "Ground states of nonlinear Schrödinger equations with potentials," Annales de l'Institut Henri Poincaré, vol. 23, no. 6, pp. 829-837, 2006. 
[8] H. Zhu, "A note on asymptotically linear Schrödinger equation on $\mathbb{R}^{N}$," Advanced Nonlinear Studies, vol. 9, no. 1, pp. 81-94, 2009.

[9] A. Ambrosetti, V. Felli, and A. Malchiodi, "Ground states of nonlinear Schrödinger equations with potentials vanishing at infinity," Journal of the European Mathematical Society (JEMS), vol. 7, no. 1, pp. 117-144, 2005.

[10] A. Ambrosetti, A. Malchiodi, and D. Ruiz, "Bound states of nonlinear Schrödinger equations with potentials vanishing at infinity," Journal d'Analyse Mathématique, vol. 98, pp. 317-348, 2006.

[11] A. Ambrosetti and Z.-Q. Wang, "Nonlinear Schrödinger equations with vanishing and decaying potentials," Differential and Integral Equations, vol. 18, no. 12, pp. 1321-1332, 2005.

[12] A. Ambrosetti and D. Ruiz, "Radial solutions concentrating on spheres of nonlinear Schrödinger equations with vanishing potentials," Proceedings of the Royal Society of Edinburgh, vol. 136, no. 5, pp. 889-907, 2006.

[13] J. Byeon and Z.-Q. Wang, "Spherical semiclassical states of a critical frequency for Schrödinger equations with decaying potentials," Journal of the European Mathematical Society (JEMS), vol. 8, no. 2, pp. 217-228, 2006.

[14] D. Bonheure and J. Van Schaftingen, "Nonlinear Schrödinger equations with potentials vanishing at infinity," Comptes Rendus Mathématique, vol. 342, no. 12, pp. 903-908, 2006.

[15] C. Liu, Z. Wang, and H.-S. Zhou, "Asymptotically linear Schrödinger equation with potential vanishing at infinity," Journal of Differential Equations, vol. 245, no. 1, pp. 201-222, 2008.

[16] J. Su, Z.-Q. Wang, and M. Willem, "Nonlinear Schrödinger equations with unbounded and decaying radial potentials," Communications in Contemporary Mathematics, vol. 9, no. 4, pp. 571-583, 2007.

[17] B. Opic and A. Kufner, Hardy-Type Inequalities, vol. 219 of Pitman Research Notes in Mathematics Series, Longman Scientific \& Technical, Harlow, UK, 1990.

[18] I. Ekeland, Convexity Methods in Hamiltonian Mechanics, vol. 19, Springer, Berlin, Germany, 1990.

[19] J. Su, Z.-Q. Wang, and M. Willem, "Weighted Sobolev embedding with unbounded and decaying radial potentials," Journal of Differential Equations, vol. 238, no. 1, pp. 201-219, 2007. 


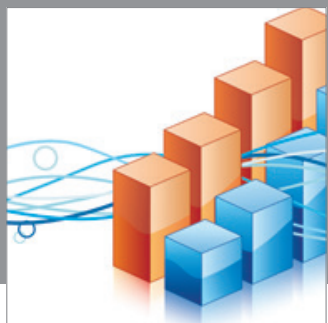

Advances in

Operations Research

mansans

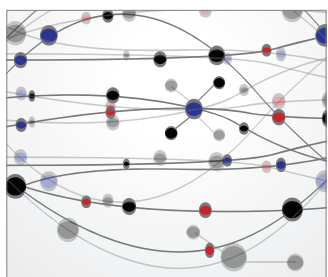

The Scientific World Journal
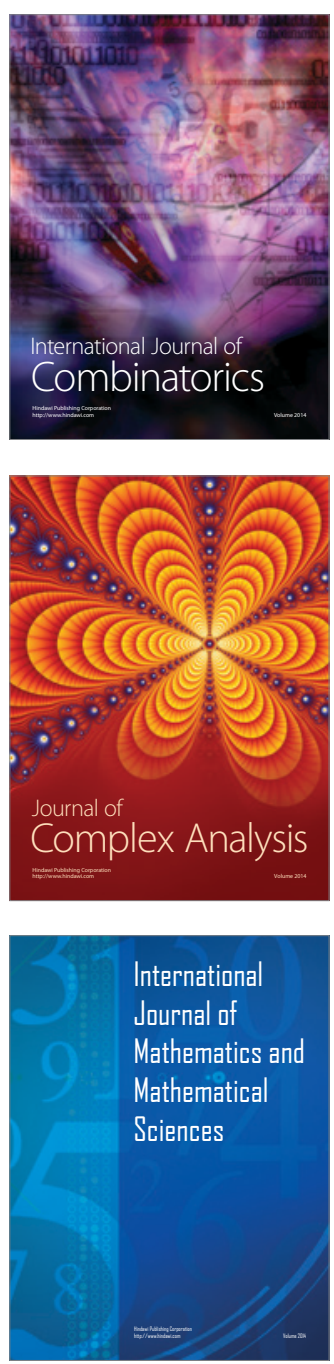
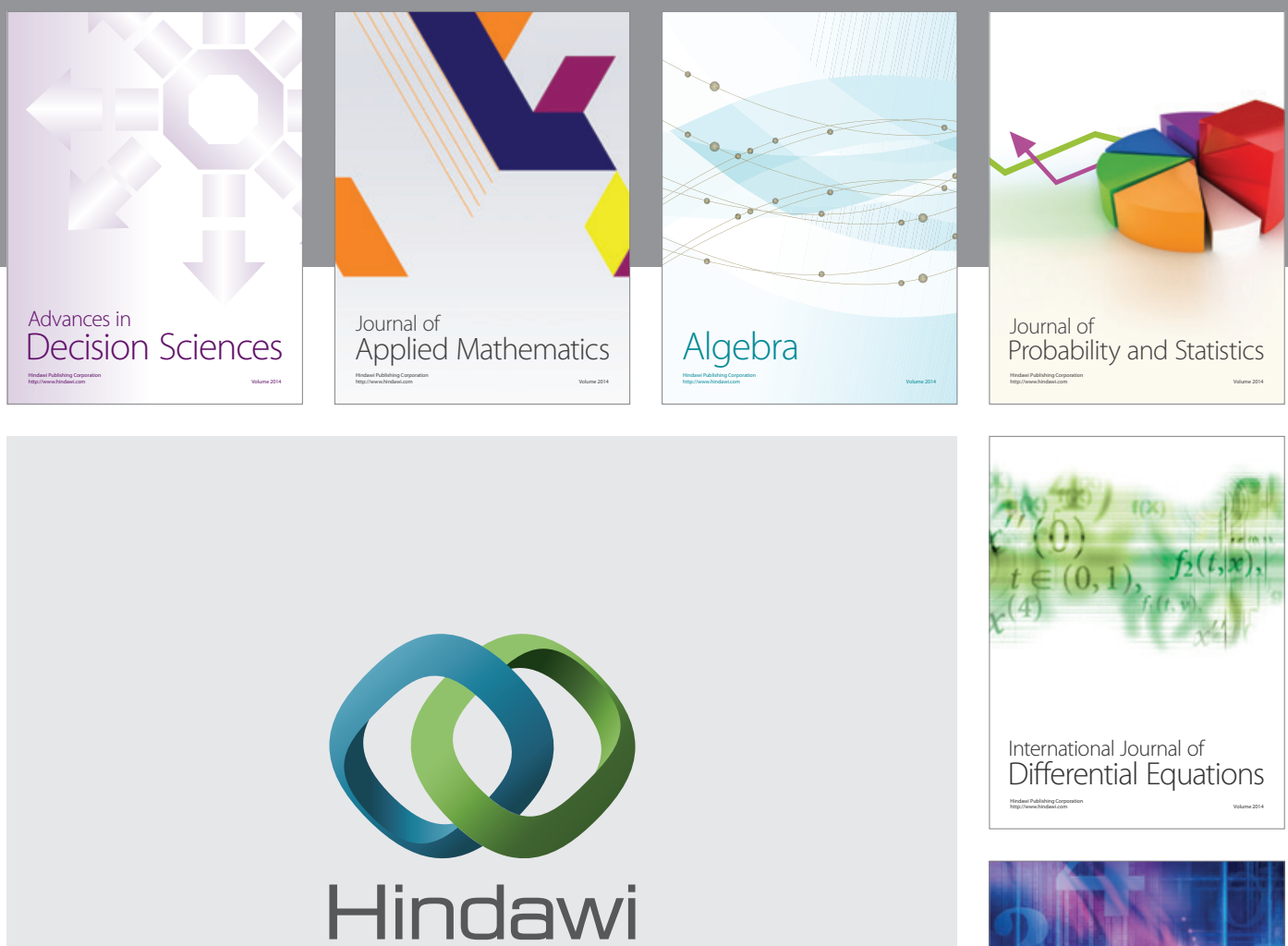

Submit your manuscripts at http://www.hindawi.com
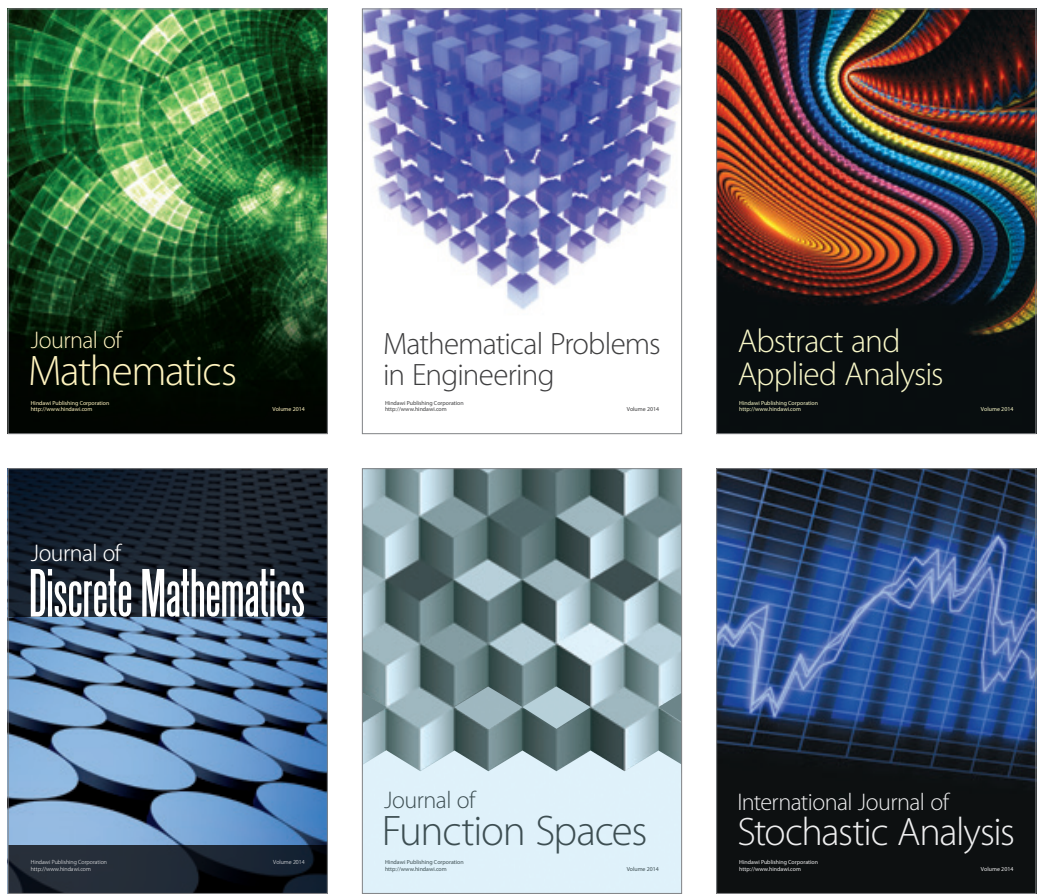

Journal of

Function Spaces

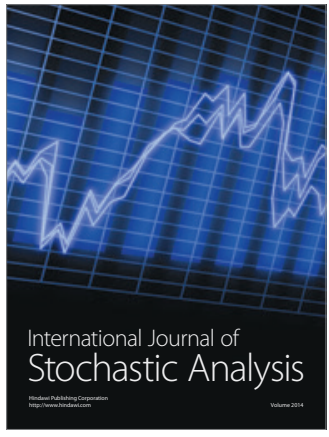

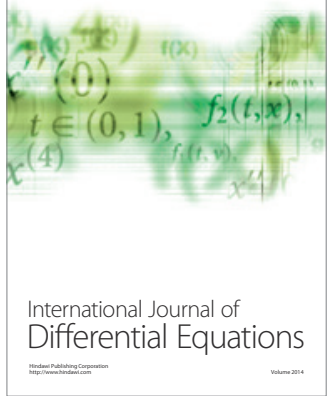
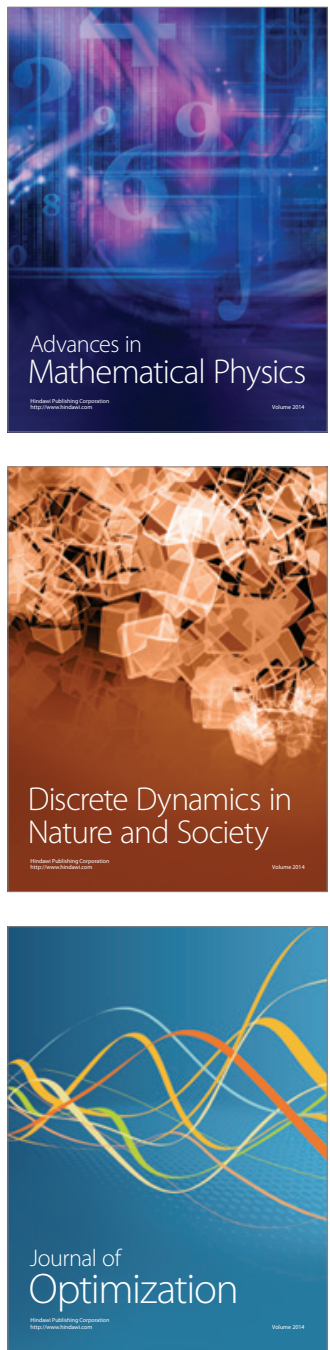